\title{
A family study of endophenotypes for psychosis within an early intervention programme in Hong Kong: Rationale and preliminary findings
}

\author{
LUI Simon S Y ${ }^{1,2,3}$, SHAM Pak ${ }^{4}$, CHAN Raymond C K ${ }^{1}$ \& CHEUNG Eric F C ${ }^{3 *}$ \\ ${ }^{1}$ Neuropsychology and Applied Cognitive Neuroscience Laboratory, Key Laboratory of Mental Health, Institute of Psychology, Chinese Academy \\ of Sciences, Beijing 100101, China; \\ ${ }^{2}$ Graduate University of Chinese Academy of Sciences, Beijing 100080, China; \\ ${ }^{3}$ Castle Peak Hospital, Hong Kong, China; \\ ${ }^{4}$ Department of Psychiatry, the University of Hong Kong, Hong Kong, China
}

Received March 12, 2011; accepted June 15, 2011; published online September 2, 2011

\begin{abstract}
The study of endophenotypes may be a viable strategy to tackle the genetic complexity and phenotypic heterogeneity of psychosis, but this research direction is relatively under-developed in China as compared to Western countries. We have recently initiated one of the first family studies of endophenotypes for psychosis in China. Patients entering an established early psychosis intervention service are recruited into this research project for phenotyping, endophenotyping and genotyping. At the endophenotypic level, four domains (neurological soft signs, neurocognition of prospective memory, social cognition of facial emotion recognition, and affective cognition of anticipatory and consummatory pleasure) are studied in the sample of patients with psychosis and their unaffected siblings. This article illustrates the benefit of a research-oriented clinical programme and its findings based on the data collected as of early 2011.
\end{abstract}

psychosis, endophenotypes, neuropsychology, social cognition

Citation: Lui S S Y, Sham P, Chan R C K, et al. A family study of endophenotypes for psychosis within an early intervention programme in Hong Kong: Rationale and preliminary findings. Chinese Sci Bull, 2011, 56: 3394-3397, doi: 10.1007/s11434-011-4734-2

\section{Development of a family study for neurocogni- tive endophenotypes and incorporating the research project into an established clinical programme}

The phenotypic heterogeneity of schizophrenia is believed to contribute to the difficulties in identifying susceptibility genes of large effect size (Odds Ratio > 1.5) [1,2]. A high level of heterogeneity is understandable in light of the uncertain biological validity of the current operational diagnostic system as well as recent evidence from psychiatric epidemiological studies which suggests that psychosis is a continuous phenotype [3]. Gottesman et al. [4-6] proposed that endophenotypes, conceptualised as markers which bridge

\footnotetext{
*Corresponding author (email: cheungfc@ ha.org.hk)
}

the gap between genes and symptoms (phenotype), may clarify the elusive disease processes of psychiatric disorders, and offer a potential solution to overcome phenotype heterogeneity in genetic studies. This endophenotype approach [6] and the recent concept of continuous phenotype [3] would require the recruitment of clinically or genetically high risk groups for studies, because these unaffected individuals may be "carriers" of vulnerable genes. Many potential endophenotypic markers are neurocognitive dysfunctions, such as working memory and attention $[7,8]$. It is possible that neurocognitive dysfunctions which are more closely related to the underlying neurobiological basis of psychosis may better predict the development of psychotic illness among high-risk individuals and the long-term clinical outcome of patients with established psychosis, than psychotic symptoms and other routine clinical parameters. It would be 
of much practical interest to clinicians if biological markers with predictive validity can be found.

Recent studies suggest that schizophrenia and bipolar disorder have closely related genetic basis [9], and therefore can be studied using the same endophenotype paradigm. To date, a limited number of research projects in Western countries have been specifically developed to address issues concerning endophenotypes, e.g. the Consortium of Genetics of Schizophrenia $[1,7,8]$, but similar projects are rare in China. Given differences in ethnicity, environment, language, and culture between Asian and Western countries, China has developed its own framework for endophenotype research, viz. the Consortium of Human Information and Neurocognitive Endophenotype (CHINE) [10].

We are currently developing a research project to specifically examine a number of candidate endophenotypes for psychosis in the Hong Kong Chinese population. In Hong Kong, a well-established early intervention programme for psychosis provides a good foundation for such a research project. The Early Assessment Service for Young People with Psychosis (EASY) in Hong Kong is a clinical programme established in 2001 [11], which aims to provide early detection and intervention for young people aged between 15 and 25 developing first-onset psychotic disorders. Patients receive care under a case management approach from the early intervention team for up to three years after the onset of psychosis, and their clinical outcome and course of illness are monitored by the same team of staff. Since its work involves a high degree of engagement of patients and their families, continuity of care, and access to a large cohort of first-onset psychosis patients who are medication-naive and free from the confounding effect of long term hospitalisation, the early intervention programme provides an excellent opportunity to study endophenotype for psychosis.

Making use of this valuable cohort of first-onset patients, a group of clinicians and scientists from Hong Kong and Beijing launched a joint research-based clinical programme in Castle Peak Hospital of Hong Kong to investigate a number of potential endophenotypic markers using a family study approach. In addition to clinical assessment (phenotyping), detailed endophenotype measures and DNA collection of both recruited patients and their first-degree relatives are part of the study paradigm. A number of neurocognitive function assessments thought to have high translational potential have been chosen as potential endophenotypes in this study while the inclusion of proteomics in this project may provide further opportunity to investigate the effect of genotype on psychosis.

The main objective of this research-based clinical programme is to examine specifically the prevalence of several neurocognitive dysfunctions in schizophrenia and bipolar disorder, which include neurological soft signs, neurocognitive parameters such as prospective memory that relate closely to clinical problems (e.g. forgetting to take medications), affective cognition such as anticipatory and consummatory pleasure, and social cognition such as facial emotional recognition. We also aim to test the endophenotypic properties of these neurocognitive dysfunctions based on the criteria proposed by Gottesman and Gould [6], i.e. (1) illness association; (2) state independence; (3) familial association; (4) co-segregation; (5) heritability. In addition, we also examine the relationship between longitudinal outcome (up to four years after onset of psychosis) and these neurocognitive endophenotypes. This research project is expected to continue for four years with a recruitment target of 100 families of patients with first-onset psychosis.

This study would recruit young patients aged 15-25 with first-onset psychotic disorders as they present to the early intervention service. As normally these patients are to be followed-up clinically at an interval of around 1-8 weeks for up to three years, the programme presents researchers with a valuable opportunity to conduct endophenotyping in a longitudinal manner at the sixth month, the first year, and the second year. This study would also recruit unaffected siblings for endophenotyping and parents for genotyping. The clinical follow-up in the outpatient clinic is an effective "contact point" for the researchers involved to recruit family members. In addition, the sample group recruited from the early intervention service is medication-naïve at service entry and is not subject to the confounding effects of longterm hospitalisation, long-term unemployment and social isolation (which may be present secondary to longstanding psychosis) on neurocognitive dysfunctions. Our subjects include not only patients with schizophrenia but also those with bipolar disorder.

\section{Preliminary findings}

As of early 2011, we have recruited 71 patients with schizophrenia, 39 patients with bipolar disorder, and 60 unaffected first-degree relatives of patients with psychosis. Using the cross-sectional data we obtained, the relationship between clinical status (i.e. schizophrenia and bipolar disorder) and prospective memory and neurological soft signs was examined. These two neurocognitive functions were also examined in genetically high-risk group and in control subjects. As longitudinal data has yet to be collected and the current sample size remains relatively small, we focus, in this article, only on illness association and familial association, and not state independence and heritability. Data on affective cognition and social cognition are not discussed in this article due to the same reasons.

\subsection{Neurological soft-signs in patients with schizophrenia and their unaffected siblings, and patients with bipolar disorder}

Empirical evidence supporting neurological soft signs (NSS) 
as a potential endophenotypic marker of psychosis has accumulated in the literature. A recent review paper summarised the previous findings and concluded that neurological soft signs are associated with schizophrenia at different stages of illness (prodromal, drug-naive, first-onset, and chronic remitted schizophrenia), and are also found in unaffected relatives of patients with schizophrenia [12]. In this study, we examined motor coordination, sensory integration, and inhibition, using the Cambridge Neurological Inventory (CNI) in our sample. The presence and severity of neurological soft signs (as indicated by the raw scores of each subset of CNI) in patients with schizophrenia and their unaffected relatives, and patients with bipolar disorder are presented in Figure 1. We found that the levels of neurological soft signs in patients with schizophrenia and bipolar disorder are similar to each other, and are higher than in unaffected siblings and healthy subjects. This is consistent with our expectation that the two phenotypes (schizophrenia and bipolar disorder) share a common potential endophenotypic marker of neurological soft signs. The level of neurological soft signs in unaffected relatives of patients with schizophrenia was moderate, while that in healthy subjects was the lowest. Our findings support the illness association property of neurological soft signs (total score) in patients with schizophrenia $[F(2,68)=13.1 ; P<0.01]$ and bipolar affective disorder $[F(2,54)=14.0 ; P<0.01]$. Moreover, NSS also seemed to have familial association property in schizophrenia because unaffected relatives of patients with schizophrenia performed more poorly than healthy subjects $[F(2,85)=13.1$; $P<0.01]$.

\subsection{Prospective memory in first-onset schizophrenia and their nonpsychotic siblings}

Prospective memory (PM) is the ability to remember to carry out intended action(s) in the future, and can be classified into event-based, time-based and activity-based PM. This ability is related to everyday functioning such as remembering to take medications and to attend a medical

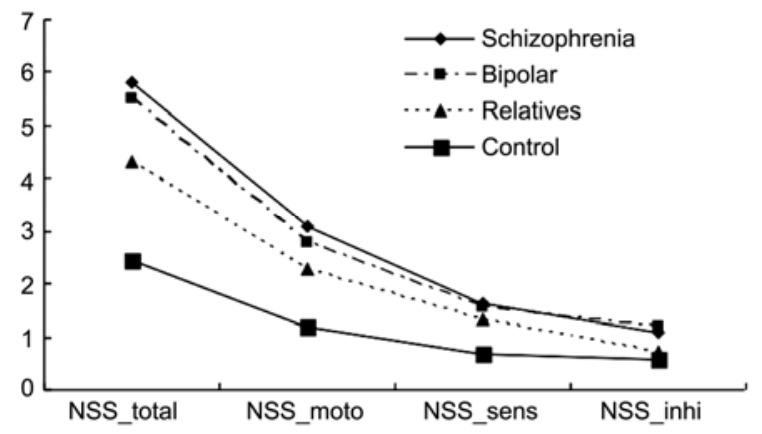

Figure 1 Neurological soft signs among patients with schizophrenia ( $n=$ $41)$, their unaffected relatives $(n=58)$, patients with bipolar disorder $(n=$ $27)$, and healthy subjects $(n=29)$. NSS_total, total score of soft signs; NSS_moto, motor coordination subscale score; NSS_sens, sensory integration subscale score; NSS_inhi, inhibition subscale score. appointment, and is therefore of high translational potential. Our research group used a dual-task behavioural paradigm [13] to assess these functions. Previous studies have found a high test-retest reliability of this PM instrument [14]. Empirical evidence has shown that PM has a distinct neural correlate in Brodmann's Area 10 (frontal pole) [15,16]. Moreover, a few studies have suggested that PM deficit is present in clinically and genetically high risk groups, e.g. in patients with schizotypal personality disorder $[13,17]$. In the light of these previous findings, we hypothesised that PM is an endophenotype of schizophrenia. The methodology of this study has been discussed elsewhere [18]. Our findings suggest that schizophrenia has significant group effect on PM performance even after other memory, attention, and executive functions were controlled for. Our findings therefore strongly support the illness association property of PM in first-onset schizophrenia (mean duration of illness: 1.7 years). Given that Wang et al. [19] found in a metaanalysis that patients with chronic schizophrenia (average duration of illness ranged from 4.7 to 26.1 years) had impaired PM function, our findings provided preliminary evidence that PM may have state-independence property regardless of illness duration.

Figure 2 shows the mean PM scores of the three groups. Although we found no significant difference in PM performance between siblings and healthy subjects, the mean PM score of the sibling group is intermediate between those of the schizophrenia group (which had the lowest PM scores) and healthy subjects (which had the highest PM scores), and a trend suggesting familial association of PM could be observed in the findings.

\section{Discussion}

To date we are only able to report preliminary findings based on a relatively small initial sample, and a larger sample is needed to investigate the familial association property of PM and NSS. Moreover, genotypic and longitudinal data are needed before it is possible to examine whether the putative endophenotypes fulfill Gottesman and Gould's [6] other criteria and their relevance to clinical outcome.

Few endophenotype research projects examine both scientific and clinical research questions with translational

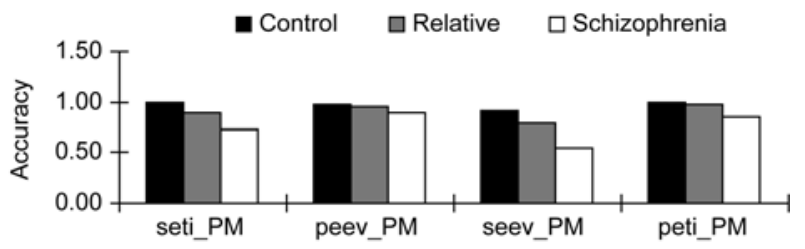

Figure 2 Prospective memory performance among the patients with schizophrenia, their first-degree siblings, and healthy subjects. Seti_PM, semantic time-based PM; peev_PM, perceptual event-based PM; seev_PM, semantic event-based PM; peti_PM, perceptual time-based PM. 
potential. The COGS (Consortium on the Genetics of Schizophrenia) and Glahn et al.'s endophenotype project [20] focus on examining candidate endophenotypes based on Gottesman's criteria but not clinical outcome. Our research-based clinical programme has the advantage of linking neurocognitive endophenotypes to clinical outcome measures and to examine their relationship. The neurocognitive measures (e.g., social cognition and prospective memory) we use in this project are believed to be more closely related to everyday functioning than measurements of traditional neurocognitive functions such as working memory, attention, and executive functions. Distinct from similar projects (such as the COGS) in Western countries, we are able to carry out endophenotyping at the drug-naive stage for most of our research subjects and on patients with bipolar disorder. Other benefits of incorporating research study into a clinical programme include the ease in subject recruitment, and the opportunity to rigorously examine the predictive properties of neurocognitive endophenotype on clinical outcomes. Moreover, it provides an opportunity for clinicians to detect subtle neurocognitive deterioration early in the course of illness and to arrange cognitive rehabilitation programmes in a timely manner, which is of potential benefit to patients.

Nevertheless, several limitations of this programme should be borne in mind. Firstly, as we recruit all subjects and their families who enter the early intervention service naturalistically, the samples contain multiple diagnostic categories and this heterogeneity may reduce the contrast in endophenotypic traits between probands and siblings needed to maximise the power of genetic analysis, as compared to the COGS. Secondly, there is limited empirical evidence in previous studies for shared endophenotypic profiles between schizophrenia and bipolar disorder, and it is uncertain whether our recruitment of both groups of diagnoses would further complicate the phenotype heterogeneity of our sample and therefore undermine the power of the endophenotype strategy. Thirdly, this project only collect behavioural data of the neurocognitive endophenotypes, but neural substrates measured by neuroimaging and event-related potentials (ERP) are thought to lie even more proximal to genes than behavioural functions. Finally, our sample size remains small to date. Because of the limit of service throughput of the early intervention service, it is expected that we cannot recruit a large sample within a short period of time. Therefore, multi-centre collaboration (such as CHINE) is clearly necessary in the future. As psychiatric research in Mainland China is blooming, we expect more sites to join CHINE and more future effort to strive for nationwide quality assurance of endophenotype data.

This work was supported by the Project-Oriented Hundred Talents Programme (O7CX031003), the Knowledge Innovation Project of the
Chinese Academy of Sciences (KSCX2-YW-R-131, KSCX2-EW-J-8), a grant from the National Science Fund for Distinguished Young Scholars (81088001) to Raymond Chan.

1 International Schizophrenia Consortium, Purcell S M, Wray N R, Stone J L, et al. Common polygenic variation contributes to risk of schizophrenia and bipolar disorder. Nature, 2009, 460: 748-752

2 Shi J, Levinson D F, Duan J, et al. Common variants on chromosome 6p22.1 are associated with schizophrenia. Nature, 2009, 460: 753

3 Linscott R J, van Os J. Systematic review of categorical versus continuum model in psychosis: Evidence for discontinuous subpopulations underlying a psychometric continuum. Implications for DSM-V, DSM-VI and DSM-VII. Annu Rev Clin Psychol, 2010, 6: 391-419

4 Gottesman I I, Shields J. Schizophrenia and Genetics: A Twin Study Vantage Point. New York: Academic Press, 1972

5 Gottesman I I, Shields J. Genetic theorizing and schizophrenia. Br J Psychiatry, 1973, 122: 15-30

6 Gottesman I I, Gould T D. The endophenotype concept in psychiatry: Etymology and strategic intentions. Am J Psychiatry, 2003, 160: 636-645

7 Braff D L, Freedman R, Schork N J, et al. Deconstructing schizophrenia: An overview of the use of endophenotypes in order to understand a complex disorder. Schizophr Bull, 2007, 33: 21-32

8 Braff D L, Greenwood T A, Swerdlow N R, et al. Advances in endophenotyping schizophrenia. World Psychiatry, 2008, 7: 11-18

9 Ivleva E I, Morris D W, Moates A F, et al. Genetics and intermediate phenotypes of the schizophrenia-bipolar disorder boundary. Neurosci Biobehav Rev, 2010, 34: 897-921

10 Chan R C K, Gottesman I I, Ge Z, et al. Strategies for the study of neuropsychiatric disorders using endophenotypes in developing countries: A potential databank from China. Front Hum Neurosci, 2008, 4: article 207

11 Chen E. Developing an early intervention service in Hong Kong. In: Ehmann T, MacEwan G W, Honer W G, eds. Best Care in Early Psychosis Intervention: Global Perspectives. London: Taylor \& Francis, 2004

12 Chan R C K, Gottesman I I. Neurological soft signs as candidate endophenotypes for schizophrenia: A shooting star or a Northern star? Neurosci Biobehav Rev, 2008, 32: 957-971

13 Wang Y, Chan R C K, Yu X, et al. Prospective memory deficits in subjects with schizophrenia spectrum disorders: A comparison study with schizophrenic subjects, psychometrically defined schizotypal subjects, and healthy controls. Schizophr Res, 2008, 106: 70-80

14 Wang Y, Chan R C K, Cui J, et al. Stability of prospective memory deficits in individuals with schizotypal personality traits. Psychiatry Res, 2011, doi:10.1016/j.psychres.2011.01.002

15 Burgess P W, Scott S K, Frith C D. The role of the rostral frontal cortex (area 10) in prospective memory: A lateral versus medial dissociation. Neuropsychologia, 2003, 41: 439-453

16 Okuda J, Fujii T, Ohtake H, et al. Differential involvement of regions of rostral prefrontal cortex (Brodmann area 10) in time- and event-based prospective memory. Int J Psychophysiol, 2007, 64: 233-246

17 Wang Y, Chan R C K, Cui J, et al. Prospective memory in non-psychotic first-degree relatives of patients with schizophrenia. Psychiatry Res, 2010, 179: 285-290

18 Lui S S Y, Wang Y, Liu A C Y, et al. Prospective memory in patients with first-onset schizophrenia and their nonpsychotic siblings. Neuropsychologia, 2011, 49: 2217-2224

19 Wang Y, Cui J, Chan R C K, et al. Meta-analysis of prospective memory in schizophrenia: Nature, extent, and correlates. Schizophr Res, 2009, 114: 64-70

20 Glahn D C, Almasy L, Blangero J, et al. Adjudicating neurocognitive endophenotypes for schizophrenia. Am J Med Genet B Neuropsychiatr Genet, 2007, 144B: 242-249

Open Access This article is distributed under the terms of the Creative Commons Attribution License which permits any use, distribution, and reproduction in any medium, provided the original author(s) and source are credited. 